\title{
Reduced eosinophil apoptosis in induced sputum correlates with asthma severity
}

\author{
C.J.A. Duncan*, A. Lawrie*, M.G. Blaylock*, J.G. Douglas", G.M. Walsh*
}

Reduced eosinophil apoptosis in induced sputum correlates with asthma severity. C.J.A. Duncan, A. Lawrie, M.G. Blaylock, J.G. Douglas, G.M. Walsh. (C)ERS Journals Ltd 2003.

ABSTRACT: This study was carried out to investigate the relationship between induced sputum eosinophil apoptosis and clinical severity score, airway obstruction and symptom scores in patients with chronic stable asthma.

Altogether, 41 chronic stable asthmatic subjects of varying severity defined by Aas score and 17 control subjects underwent spirometry, symptom questionnaire and successful sputum induction. Sputum was processed and cytospins prepared for light microscopy to determine normal and apoptotic eosinophils.

Mild asthmatic subjects had a significantly lower percentage sputum eosinophils and a significantly higher eosinophil apoptotic ratio (AR) than moderate or chronic severe asthmatics. Severe asthmatic subjects had a significantly greater age, duration of asthma and sputum eosinophil count $\cdot \mathrm{mL}^{-1}$ than mild asthmatic subjects. Asthmatic subjects' symptom scores, severity scores and age inversely correlated with AR and the percentage of sputum eosinophils. Baseline forced expiratory volume in one second inversely correlated with percentage sputum eosinophils and positively correlated with AR.

The study demonstrates a relationship between reduced sputum eosinophil apoptosis and increased clinical severity of chronic stable asthma, providing additional evidence that eosinophil apoptosis may be important in the resolution of esinophilic airway inflammation in asthma.

Eur Respir J 2003; 22: 484-490.
*Allergic and Asthmatic Inflammation Research Group, Dept of Medicine and Therapeutics, Institute of Medical Sciences, University of Aberdeen Medical School, and ${ }^{\#}$ Respiratory Medicine Unit, Grampian University National Health Service Trust, Aberdeen, UK.

Correspondence: G.M. Walsh, Dept of Medicine and Therapeutics, Institute of Medical Sciences Building, University of Aberdeen, Foresterhill, Aberdeen, AB25 2ZD, UK. Fax: 441224555766

E-mail: g.m.walsh@abdn.ac.uk

Keywords: Airway inflammation, apoptosis, asthma, eosinophils, severity of illness index, sputum

Received: November 282002

Accepted after revision: April 82003

C.J.A. Duncan and A. Lawrie contributed equally to this work.
Inappropriate accumulation of eosinophils and the subsequent release of their potent armoury of mediators, including cytotoxic granule proteins, lipid mediators, cytokines and chemokines, are thought to contribute significantly to the airway inflammation underlying asthma pathogenesis [1]. Apoptosis (programmed cell death) is fundamental to a viable immune system [2] and vital for the resolution of inflammation. Cells are rapidly phagocytosed either whole or as fragments bound by an intact membrane, preventing exposure of adjacent tissues to their intracellular contents. An evolving hypothesis that the tissue load of eosinophils in allergic disorders and asthma is related to the inhibition of or defects in the apoptotic process is supported by studies that provide evidence that eosinophil clearance by apoptosisinduction has clinical relevance in asthma [3-5]. Therefore, this may represent a plausible target for novel therapeutic intervention.

Examination of induced sputum using nebulised hypertonic saline is a well-validated noninvasive procedure for the assessment of airway inflammation in asthma [5-8]. However, to date there have been few studies that have utilised this technique to compare the relationship between measures of eosinophil apoptosis or survival in induced sputum with clinical and functional parameters of disease severity [5-10]. The authors hypothesised that there is a relationship between reduced eosinophil apoptosis and both a global clinical severity score and separate components of the asthma phenotype. The aim of the current study was to assess eosinophil apoptosis in induced sputum samples from clinically stable asthmatic individuals in order to investigate the relationship between eosinophil apoptosis and clinical severity, airway obstruction and symptom scores.

\section{Methods}

\section{Study subjects}

Altogether, 48 subjects with clinically stable asthma of varying severity (aged 17-67 yrs) were randomly recruited from the Respiratory Outpatient Department of Aberdeen Royal Infirmary. Healthy control subjects $(n=21)$, aged 20-56 yrs, were recruited from staff at the Respiratory Outpatient Department. No information on airway inflammatory parameters prior to the start of the study was available for either patients or control subjects. Diagnosis of asthma was defined according to American Thoracic Society criteria [11]. All subjects underwent reversibility testing to $200 \mu \mathrm{g}$ of salbutamol. All asthma patients had previously exhibited reversibility at some time, defined as an increase in forced expiratory volume in one second (FEV1) of $>15 \%$ predicted following salbutamol. Subjects were excluded from the study if they had a respiratory infection in the month preceding the study. Asthmatic subjects were also excluded if they had an exacerbation in the preceding month, or a postbronchodilator FEV1 of $<50 \%$ pred. All asthmatic subjects continued on their normal maintenance 
medication, including inhaled corticosteroids (ICS), and no subjects received oral corticosteroid or antibiotic therapy in the month preceding the study. Asthmatic subjects had no clinical or radiological evidence of other respiratory disease. All subjects were non or exsmokers for $\geqslant 1 \mathrm{yr}$ prior to inclusion. All subjects gave written informed consent and the Joint Ethics Committee of the Grampian Health Board approved the study. The pre-study characteristics of all subjects are shown in table 1 . The median age and duration of disease of subjects with severe asthma (Aas 4-5) was significantly higher than those with mild (Aas 1-2) and moderate (Aas 3) disease. There were no significant differences between ICS dose in mild, moderate and severe groups.

\section{Study design}

A single-centre cross-sectional observational study design was used. All subjects attended an initial clinical assessment, at which symptoms were assessed. Details of asthma duration, current medication and smoking history were recorded from patient interviews and clinical notes, Aas scores were calculated, and venepuncture performed for peripheral blood eosinophil count and specific immunoglobulin (Ig)E levels. This was followed by a return visit within 2 weeks for spirometry and sputum induction.

\section{Methods}

At initial visit symptoms of cough, wheeze, dyspnoea, chest tightness and sputum production were assessed, and the patient completed part 1 of the validated St George's Respiratory Questionnaire that assesses symptom frequency over the previous year [12]. Weighted scores for each response were added and the final score was calculated as a percentage of the total possible score. Clinical severity was assessed using the validated Aas score as described previously [13]. In addition, $15 \mathrm{~mL}$ blood was taken for peripheral blood eosinophil count (Advia ( 120 Haematology System; Bayer Diagnostics, NY, USA) and assessment of atopy. Atopic status was defined by the presence of specific $\operatorname{IgE}$ antibodies $\left(\geqslant 0.35 \mathrm{U} \cdot \mathrm{L}^{-1}\right)$ to at least one common inhaled allergen by the catabolic gene activator protein method (Pharmacia-Upjohn, Uppsala, Sweden) [14]. On a subsequent visit, spirometry was performed using an electronic spirometer (Compact II; Vitalograph, Buckinghamshire, UK). The response to the bronchodilator was assessed $15 \mathrm{~min}$ after administration of $200 \mu \mathrm{g}$ of salbutamol via a metered-dose inhaler. Sputum induction was performed according to the method of PIN et al. [15] using an ultrasonic nebuliser (Sonix 2000; Medix, Lutterworth, UK). Briefly, sputum induction was performed using $5 \mathrm{~mL}$ of hypertonic $(3 \%)$ saline solution administered via the nebuliser for 5-min periods over a total duration of $30 \mathrm{~min}$. The concentration was increased in $1 \%$ increments from $3-5 \%$ at intervals of $10 \mathrm{~min}$. Subjects were encouraged to expectorate sputum at any time throughout the procedure after rinsing the mouth and throat carefully. FEV1 was measured every $5 \mathrm{~min}$ and the procedure was stopped if FEV1 fell $<20 \%$ of the postbronchodilator value or troublesome symptoms occurred. Sputum samples were transferred to the laboratory on ice as soon as possible, in all cases within $1 \mathrm{~h}$. Sputum processing was performed as described previously

Table 1.-Characteristics of study population

\begin{tabular}{|c|c|c|c|c|c|}
\hline & \multicolumn{4}{|c|}{ Asthma } & \multirow[t]{2}{*}{ Control } \\
\hline & Mild & Moderate & Severe & Total & \\
\hline Aas score & $1-2$ & 3 & $4-5$ & & \\
\hline Subjects $n$ & 30 & 8 & 10 & 48 & 21 \\
\hline \multicolumn{6}{|l|}{ Age yrs } \\
\hline Median (IQR) & $37.5(22-50)$ & $37(30-54)$ & $52(39.5-56)^{\#}$ & $43(29-54)$ & $32(27-38)$ \\
\hline Range & $17-59$ & $22-67$ & $32-62$ & $17-67$ & $20-56$ \\
\hline Sex Male/Female & $10 / 20$ & $3 / 5$ & $5 / 5$ & $18 / 30$ & $7 / 14$ \\
\hline \multicolumn{6}{|l|}{ Smoking status n $(\%)$} \\
\hline Never-smoker & $25(83)$ & $5(63)$ & $7(70)$ & 37 (77) & $17(81)$ \\
\hline Exsmoker & $5(17)$ & $3(37)$ & $3(30)$ & $11(23)$ & $4(19)$ \\
\hline \multicolumn{6}{|l|}{ Age at onset of asthma yrs } \\
\hline Median (IQR) & $15(8-26)$ & $16.5(10-37)$ & $16.5(10-20)$ & $16.5(8-29)$ & \\
\hline Range & $1-51$ & $8-47$ & $1-30$ & $1-51$ & \\
\hline \multicolumn{6}{|l|}{ Asthma duration yrs } \\
\hline Median (IQR) & $17(8-27)$ & $18(13-22)$ & $28.5(23-37)^{\top}$ & $18.5(12-29)$ & \\
\hline Range & $1-49$ & $8-34$ & $12-59$ & $1-59$ & \\
\hline \multicolumn{6}{|l|}{ Inhaled steroid dose $\mu \mathrm{g} \cdot \mathrm{day}^{-1+}$} \\
\hline Mean \pm SEM & $1948 \pm 210$ & $2200 \pm 421$ & $2560 \pm 514$ & $2117 \pm 182$ & \\
\hline Range & $200-4000$ & $800-4000$ & $800-6000$ & $200-6000$ & \\
\hline \multicolumn{6}{|l|}{ Baseline FEV1 \% pred } \\
\hline Mean \pm SEM & $83.7 \pm 3.2$ & $78.8 \pm 6.9$ & $62.4 \pm 5.1^{f}$ & $78.5 \pm 2.8$ & $98.8 \pm 2.8^{\S}$ \\
\hline Range & $75-125$ & $51-107$ & 39-93 & $39-125$ & $72-120$ \\
\hline Sputum induction success rate $\mathrm{n}(\%)$ & $27(90)$ & $6(75)$ & $8(80)$ & $41(85)$ & $17(81)$ \\
\hline \multicolumn{6}{|l|}{ Atopic status n $(\%)$} \\
\hline Atopic & $22(81)$ & $3(50)$ & $5(63)$ & $30(73)$ & $7(41)$ \\
\hline Nonatopic & $5(19)$ & $3(50)$ & $3(37)$ & $11(27)$ & $10(59)$ \\
\hline \multicolumn{6}{|l|}{ Blood eosinophils $\times 10^{9} \cdot \mathrm{L}^{-1}$} \\
\hline Mean \pm SEM & $0.48 \pm 0.08$ & $0.36 \pm 0.08$ & $0.94 \pm 0.25^{\# \#}$ & $0.56 \pm 0.08$ & $0.18 \pm 0.03^{\text {ตฯ }}$ \\
\hline Range & $0.04-1.67$ & $0.14-0.64$ & $0.16-2.23$ & $0.04-2.23$ & $0-0.52$ \\
\hline
\end{tabular}

IQR: interquartile range; FEV1: forced expiratory volume in one second; $\%$ pred: $\%$ predicted. ${ }^{\#}: \mathrm{p}<0.05$; severe versus mild $(95 \%$ confidence interval (95\% CI): 1-22); ${ }^{\top}: \mathrm{p}<0.05$; severe versus moderate (95\% CI: 2-27); ${ }^{+}$: Inhaled corticosteroid (beclomethasone) where $1 \mu \mathrm{g}$ beclomethasone=1 $\mu \mathrm{g}$ budesonide $=0.5 \mu \mathrm{g}$ fluticasone; ${ }^{f}: \mathrm{p}<0.005$; severe versus mild $(95 \% \mathrm{CI}: 8.7-34) ;{ }^{\S}: \mathrm{p}<0.0001 ;$ asthma versus control $(95 \% \mathrm{CI}: 11.2-29.4) ;{ }^{\# \#}: \mathrm{p}<0.05$; severe versus mild (95\% CI: 0.06-0.86); ${ }^{\bullet}: \mathrm{p}<0.005$; asthma versus control $(95 \% \mathrm{CI}: 0.15-0.62)$. 
[16]. Briefly, sputum was selected from saliva, weighed and dispersed using four volumes of $0.1 \%$ dithiothreitol (Sputalysin; Calbiochem-Novabiochem UK Ltd, Nottingham, UK). After $15 \mathrm{~min}$ of gentle rocking at room temperature the suspension was filtered through $48 \mu \mathrm{m}$ gauze. Squamous cell contamination $(\%)$, total cell count and total eosinophil count $\left(10^{5} \mathrm{~mL}\right)$ were obtained using a Neubauer haemocytometer (Fisher Scientific UK Ltd, Loughborough, UK) and cell viability was assessed using the trypan blue exclusion method. The cell suspension was adjusted to $1 \times 10^{6}$ cells $\cdot \mathrm{mL}^{-1}$ and $100 \mu \mathrm{m}$ of cell suspension was used to prepare cytospins at 450 revolutions per minute for $5 \mathrm{~min}$ using a Shandon cytocentrifuge (Shandon Southern Instruments, Szwickley, PA, USA). Cytospins were stained with Kimura and haematoxylin and two examiners counted 400 nonsquamous cells overall. The percentages were then averaged to give the final eosinophil counts. In addition, 200 eosinophils were counted under oil immersion and their morphology characterised as normal or apoptotic. Apoptotic eosinophils were differentiated on the basis of nuclear condensation, nuclear coalescence (shift from bilobed to monolobed nucleus) and cytoplasmic shrinkage as described previously [5]. The apoptotic ratio (AR) was calculated according to a previously validated method [5]:

$$
\% \text { apoptotic eosinophils }
$$

$$
\% \text { apoptotic eosinophils }+\% \text { eosinophils }
$$

The validity of Kimura staining was assessed against a representative sample $(n=31)$ of additional cytospin slides stained with Diff-Quik (methylene blue and eosin, Rapi-Diff II; Diachem, Burscough, UK), counted as described above. Activated eosinophil numbers were assessed using immunostaining of cytospins with specific monoclonal antibody to EG2 (Pharmacia, Uppsala, Sweden), together with a positive control (isolated peripheral blood eosinophils activated by overnight incubation with granulocyte macrophage colony stimulating factor) and a suitable isotype-matched negative control. Positive staining was visualised using the alkaline phosphatase antialkaline phosphatase (APAPP) method. All counts were performed on coded cytospins by investigators blinded to the clinical characteristics of the subjects.

\section{Analysis}

Descriptive statistics were obtained for demographic and sputum data. The differences between groups were examined using the Mann-Whitney U-test for nonparametric data and the independent unpaired t-test for parametric data. Asthmatic subjects were divided into three groups by Aas score to analyse the differences between demographic and sputum data (Aas scores 1-2 (mild), Aas score 3 (moderate), and Aas score 4-5 (severe)). Asthmatic subjects were also divided into high dose ICS (beclomethasone dipropionate $\geqslant 1000 \mu \mathrm{g}$, budesonide dipropionate $\geqslant 1000 \mu \mathrm{g}$ or fluticasone propionate $\geqslant 500 \mu \mathrm{g}$ ) and low dose ICS groups to assess the influence of daily ICS medication on the analysis. The Spearman rank correlation coefficient $(R \mathrm{~s})$ and the Pearson correlation coefficient $(r)$ were calculated to assess correlations between nonparametric and parametric data, respectively. The Kendall correlation coefficient $(\tau)$ was used to assess correlations with the ordinal Aas score. The validity of Kimura staining was expressed as the intraclass correlation coefficient (ICC) of between-method differences. All data was expressed as mean \pm SEM or median (interquartile range), with $95 \%$ confidence intervals and p-value where appropriate. A p-value $<0.05$ was considered statistically significant.

\section{Results}

\section{Sputum induction and processing}

Altogether, 41 (85\%) asthmatic subjects and 17 (81\%) controls produced a satisfactory sputum sample (table 1). The Kimura staining technique resulted in green eosinophil cytoplasmic and blue nuclear staining, and both normal and apoptotic eosinophils were identified without difficulty (fig. 1a and b). Agreements of counts between Kimura and Diff-Quik were high for both percentage sputum eosinophils and AR (ICC $=0.86,0.88$ ). Due to low cell numbers in sputum from control subjects, AR counts were determined for available cell numbers. Sputum data are summarised in table 2. Median percentage sputum eosinophils, total eosinophil count $\cdot \mathrm{mL}^{-1}$ and percentage $\mathrm{EG} 2+$ eosinophils were significantly higher $(\mathrm{p}<0.0001)$ in the asthmatic group, and mean AR was significantly lower $(\mathrm{p}<0.0001)$ in the asthmatic group than the control group. The mild asthmatic group had a lower median percentage of sputum eosinophils, and a higher mean AR than both moderate $(\mathrm{p}<0.05, \mathrm{p}<0.05)$ and severe $(\mathrm{p}<0.0005, \mathrm{p}<0.005)$ groups. The mild asthmatic group had a significantly lower median total eosinophil count $\cdot \mathrm{mL}^{-1}$ than the severe asthmatic group $(\mathrm{p}<0.05)$. Eosinophil parameters were similar between asthmatics on high $(n=32)$ and
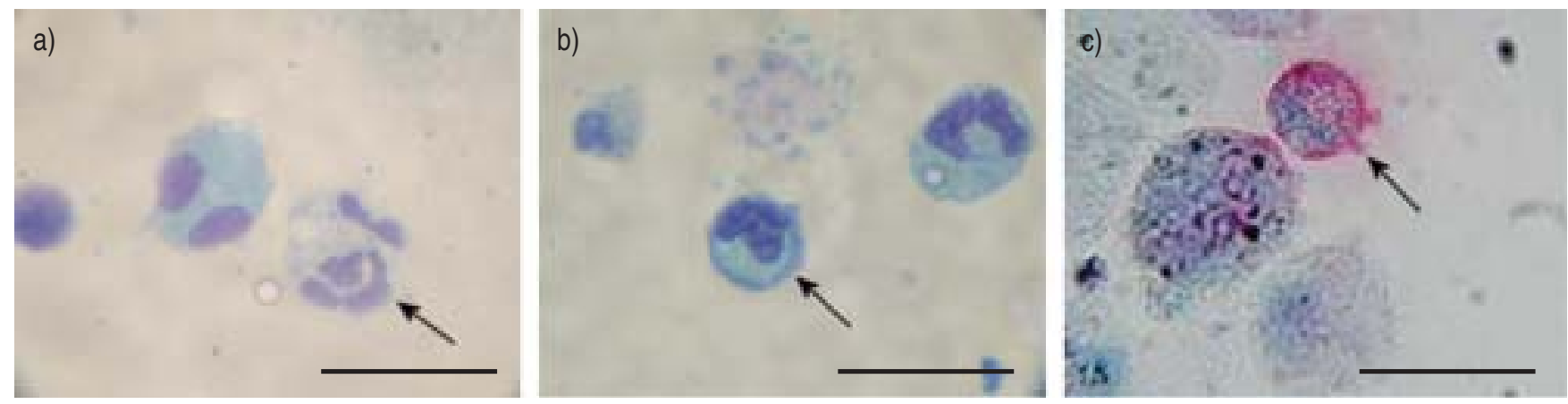

Fig. 1.- Representative oil-immersion photomicrographs of a) Kimura and haematoxylin stained cytospin demonstrating green cytoplasmic staining of a morphologically normal bilobed sputum eosinophil and the lack of green cytoplasmic staining of a morphologically normal sputum neutrophil (arrowed), b) Kimura and haematoxylin stained cytospin demonstrating the morphological characteristics of an apoptotic (arrowed) and a normal sputum eosinophil and c) EG2+ sputum eosinophil (arrowed) following alkaline phosphatase antialkaline phosphatase immunocytochemistry, demonstrating the appearance of an activated eosinophil adjacent to a pulmonary macrophage. Scale bar corresponds to $20 \mu \mathrm{m}$. 
Table 2.-Comparison of the sputum parameters between the asthmatic and control groups

\begin{tabular}{|c|c|c|c|c|c|}
\hline & \multicolumn{4}{|c|}{ Asthma } & \multirow[t]{2}{*}{ Control } \\
\hline & Mild & Moderate & Severe & Total & \\
\hline Aas Score & $1-2$ & 3 & $4-5$ & & \\
\hline Subjects $n$ & 27 & 6 & 8 & 41 & 17 \\
\hline Viability \% & $57.4 \pm 3.5$ & $62 \pm 9.7$ & $71.6 \pm 5.1$ & $61.2 \pm 3.0$ & $63.6 \pm 3.7$ \\
\hline Nonsquamous cells $\times 10^{5} \cdot \mathrm{mL}^{-1}$ & $6.1 \pm 0.9$ & $6.4 \pm 1.8$ & $5.7 \pm 1.8$ & $6.1 \pm 0.72$ & $1.93 \pm 0.52^{\#}$ \\
\hline Total eosinophils $\times 10^{5} \cdot \mathrm{mL}^{-1}$ & $0.55(0.25-1.0)$ & $0.88(0.84-1.2)$ & $2.24(0.84-4.18)^{\bullet}$ & $0.8(0.4-1.2)$ & $0.02(0-0.05)^{+}$ \\
\hline Squamous contamination $\%$ & $3.1(1.6-8.9)$ & $10.9(4.4-13.7)$ & $1.9(0.6-9.7)$ & $3.1(1.5-10.0)$ & $6.9(3.1-25.0)$ \\
\hline Sputum eosinophils $\%$ & $7.4(3.5-24.7)$ & $34.5(16.6-38.8)^{\S}$ & $50.5(46.5-62.0)^{f}$ & $20.4(6.5-44)$ & $2(0.5-3.0)^{\# \#}$ \\
\hline AR & $0.80(0.65-0.94)$ & $0.62(0.36-0.68)^{\bullet ⿻}$ & $0.49(0.13-0.56)^{++}$ & $0.69(0.49-0.89)$ & $0.98(0.97-0.99)^{\S \S}$ \\
\hline EG2+ve eosinophils \% & $31(22.6-43.3)$ & $26.5(22.8-33.0)$ & $31(26.3-38.0)$ & $31(22.0-40.5)$ & $1(0-6.0)^{f f}$ \\
\hline
\end{tabular}

Data presented as mean \pm SEM or median (IQR) unless otherwise stated. AR: apoptotic ratio. ${ }^{\#}: \mathrm{p}<0.0001$, asthma versus control $(95 \%$ confidence interval (95\% CI) 1.8-6.5); ${ }^{\uparrow}: \mathrm{p}<0.05$, severe versus mild $(95 \%$ CI $0.28-3.44) ;{ }^{+}: \mathrm{p}<0.0001$, asthma versus control $(95 \%$ CI $0.47-1.0)$; ${ }^{\circledR}: \mathrm{p}<0.05$, moderate versus mild $(95 \%$ CI $11.0-36.0) ;{ }^{f}: \mathrm{p}<0.0005$, severe versus mild $(26.6-48.8) ;{ }^{\# \#}$ : $\mathrm{p}<0.0001$, asthma versus control $(95 \%$ CI $5.5-35.0)$; ${ }^{-1}$ : $\mathrm{p}<0.05$, moderate versus mild $(95 \%$ CI $0.032-0.395) ;{ }^{+}: \mathrm{p}<0.005$, severe versus mild (CI $\left.0.21-0.57\right) ;{ }^{\$ s}: \mathrm{p}<0.0001$ asthma versus control $(95 \%$ CI $0.146-0.414) ;{ }^{f f}: \mathrm{p}<0.0001$ asthma versus control $(95 \%$ CI $20.2-33.9)$.

low $(n=9)$ dose ICS therapy. Neither percentage sputum eosinophils ( $7.4 \%$ difference, $\mathrm{p}=0.47)$, AR ( 0.15 difference, $\mathrm{p}=0.19)$ or peripheral blood eosinophils (0.33 difference, $\mathrm{p}=0.26$ ) were significantly different between the groups.

\section{Associations}

Percentage sputum eosinophils in asthmatics positively correlated with symptom score $\left(R_{\mathrm{s}}=0.72, \mathrm{p}<0.0001\right)$, Aas score $(\tau=0.58, \mathrm{p}<0.0001)$ and age $(R \mathrm{~s}=0.54, \mathrm{p}<0.001)$, and inversely correlated with baseline FEV1 $(R \mathrm{~s}=-0.46, \mathrm{p}<0.005)$ (fig. 2). AR in asthmatics inversely correlated with symptom score $(R \mathrm{~s}=-0.75, \mathrm{p}<0.0001)$, Aas score $(\tau=-0.57, \mathrm{p}<0.0001)$ and age $(R \mathrm{~s}=-0.56, \mathrm{p}<0.001)$, and positively correlated with baseline FEV1 $(R \mathrm{~s}=0.51, \mathrm{p}<0.001)$ (fig. 3). No relationship was observed between EG2 expression and either baseline FEV1 $(R \mathrm{~s}=0.08, \mathrm{p}=0.63)$, symptom score $(R \mathrm{~s}=0.24 ; \mathrm{p}=0.12)$ or Aas score $(\tau=0.05, \mathrm{p}=0.67)$. Peripheral blood eosinophils inversely correlated with AR and positively correlated with percentage sputum eosinophils $(R \mathrm{~s}=-0.57, \mathrm{p}<0.001 ; R \mathrm{~s}=0.61$, $\mathrm{p}<0.0001)$. Correlations also existed between peripheral blood eosinophil count and baseline FEV1 ( $r=-0.39, \mathrm{p}=0.03)$, Aas score $(\tau=0.33, \mathrm{p}=0.008)$ and symptom score $(R \mathrm{~s}=0.57$, $\mathrm{p}=0.0002$ ). There was no significant association between ICS dose and either AR, percentage sputum eosinophils or peripheral blood eosinophils $(R \mathrm{~s}=-0.1, \mathrm{p}=0.48 ; R \mathrm{~s}=0.1, \mathrm{p}=0.54$; $r=0.24, \mathrm{p}=0.16$ ).

\section{Discussion}

This study has demonstrated that reduced eosinophil apoptosis and increased sputum eosinophilia both significantly correlate with asthma severity, as determined by the Aas score, in patients with clinically stable disease. In addition, this study has shown that reduced eosinophil apoptosis and sputum eosinophil load are significantly correlated with the age, degree of self-reported symptoms and FEV1 restriction in these patients.

Eosinophil removal from airway tissue is thought to be dependent upon their death by the tightly controlled mechanism of apoptosis, or programmed cell death, followed by their recognition and phagocytosis by macrophages [17] or resident bronchial epithelial cells [18]. Therefore, it is of considerable interest that the factors involved in eosinophil clearance and delayed eosinophil apoptosis have been proposed to make a significant contribution to the increased tissue load of eosinophils in the asthmatic lung [19]. Luminal entry into the airway has recently been proposed as a mechanism for eosinophil clearance [20]. However, luminal entry as a sole mechanism for eosinophil disposal could be envisaged as being pro-inflammatory, since apoptotic eosinophils would lose membrane integrity and release pro-inflammatory products if not cleared from the lungs. Removal of eosinophil infiltrates via apoptosis and concomitant phagocytosis offers considerable potential to ameliorate asthmatic inflammation $[3,5]$.

While a number of studies have investigated eosinophil apoptosis in induced sputum from asthmatic subjects, this is the first report to investigate the relationship between sputum eosinophil apoptosis and measures of lung function, clinical symptom score, and global asthma severity score among chronic stable asthmatics. The findings in the current study that eosinophil AR correlates with FEV1 is in agreement with the study by JANG et al. [7], which investigated the relationship between eosinophil apoptosis and airway obstruction in patients with acute severe asthma, in which bcl-2 expression (a marker of reduced eosinophil apoptosis) inversely correlated with FEV1:forced vital capacity. In addition, VIGNOLA et al. [4] demonstrated that the proportions of apoptotic eosinophils in bronchial biopsies from asthmatic subjects were inversely correlated with the Aas score. Taken together with the results from the current study, these data indicate a relationship in asthma between the proportion of apoptotic eosinophils in the lung and both a global score of severity and separate components of the asthma phenotype (airflow obstruction and symptoms), which further indicates that eosinophil apoptosis may be important in the resolution of airway eosinophilia in asthma. In addition, WoolLEY et al. [5] demonstrated an increase in the sputum eosinophil AR and a decrease in sputum eosinophil numbers following corticosteroid treatment of patients with induced asthma exacerbation. Therefore, delayed eosinophil apoptosis may be an important cause of the increased tissue load of eosinophils seen in asthma $[9,10]$. However, a recent study by GIBSON et al. [8] investigating the anti-inflammatory effects of a single high dose of inhaled budesonide reported that a subsequent decrease in the percentage sputum eosinophils following treatment was not accompanied by an increase in the proportion of apoptotic eosinophils. The reasons for the discrepancy between this study and previous findings are unclear, but emphasise that other factors are important in determining eosinophil load in the airways, including 

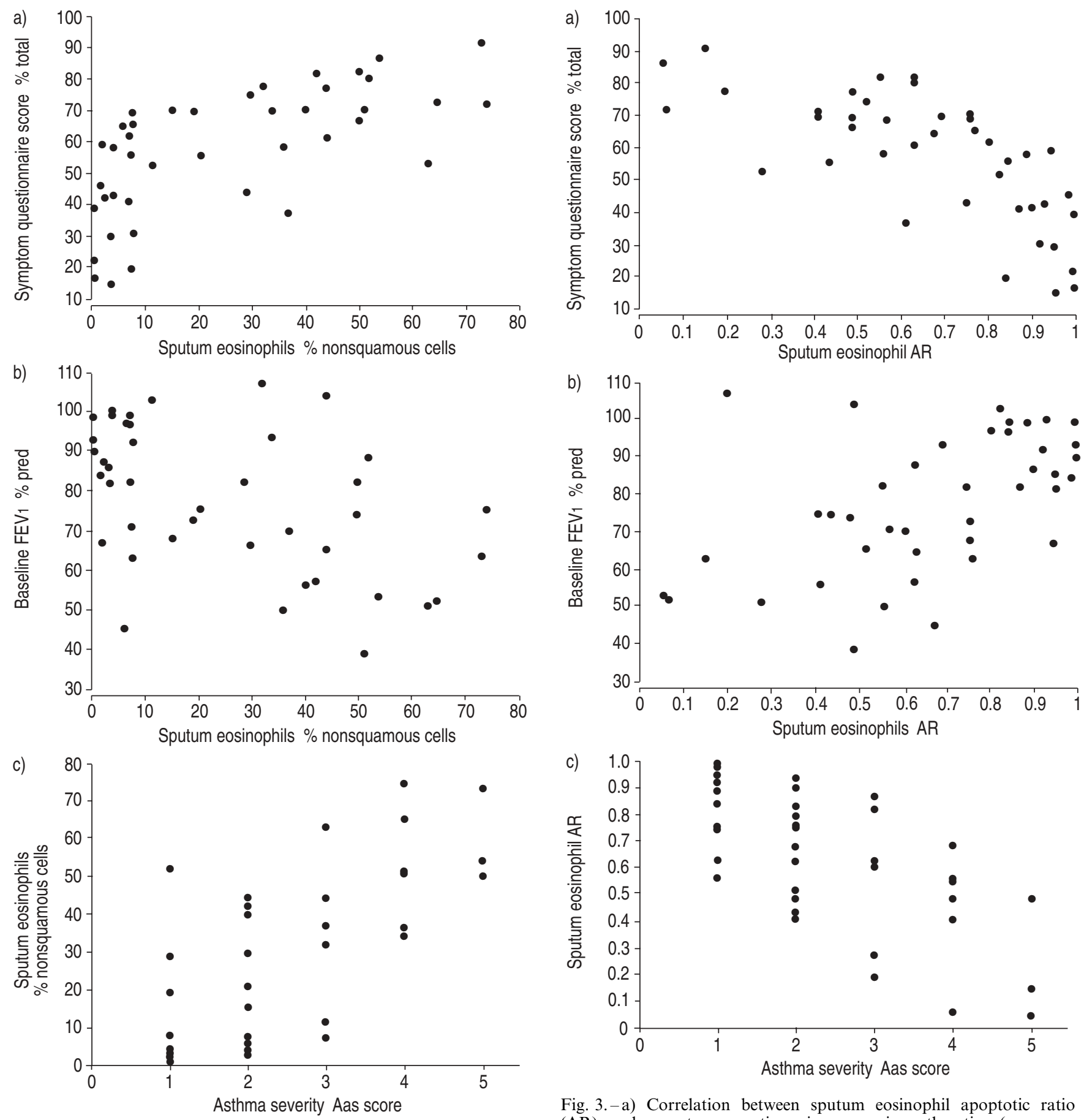

Fig. 2. - a) Correlation between sputum eosinophils and symptom questionnaire score in asthmatics (spearman correlation; $R_{\mathrm{s}}=0.72$, $\mathrm{p}<0.0001)$. b) Correlation between sputum eosinophils and baseline lung function $(\%$ predicted forced expiratory volume in one second (FEV1) prior to administration of $\beta_{2}$-agonist $)$ in asthmatics $\left(R_{s}=-0.46\right.$, $\mathrm{p}<0.005)$. c) Correlation between sputum eosinophils and asthma severity assessed by the Aas Score (Kendall correlation; $\tau=0.58$, $\mathrm{p}<0.0001$ )

augmented eosinophil production in the bone marrow, their increased release into the circulation followed by selective accumulation, and enhanced survival in the airways [3, 21].

No association was demonstrated between ICS dose and eosinophilic inflammatory parameters in the current study, particularly eosinophil:apoptotic. This may be explained by the fact that there were no significant differences between the ICS dose in subjects classified by Aas score as mild, moderate

Fig. 3.-a) Correlation between sputum eosinophil apoptotic ratio (AR) and symptom questionnaire score in asthmatics (spearman correlation; $R_{\mathrm{s}}=-0.75$, $\left.\mathrm{p}<0.0001\right)$. b) Correlation between sputum eosinophil AR and baseline lung function $(\%$ predicted forced expiratory volume in one second $\left(\mathrm{FEV}_{1}\right)$ prior to administration of $\beta_{2}$-agonist $)$ in asthmatics $\left(R_{\mathrm{s}}=0.51, \mathrm{p}<0.001\right)$. c) Correlation between sputum eosinophil AR and asthma severity assessed by the Aas score (Kendall correlation; $\tau=-0.57, \mathrm{p}<0.0001$ ).

and severe, since even those classified as mild were receiving high ICS doses. Therefore, the authors are unable to exclude the possibility that ICS treatment had an effect on the inflammatory parameters in these patients. Sputum eosinophilia was a feature of the majority of patients in this study, a finding that has been reported by other groups. For example, WARDLAW et al. [22] reported a wide variation in percentage sputum eosinophils in a study of 99 patients, with a high percentage of eosinophils observed in several patients with 
mild-to-moderate disease receiving ICS medication, as well as those admitted to hospital with acute severe asthma. Furthermore, LouIS et al. [23] studied induced sputum in 74 asthmatic and 22 control subjects with disease severity classified according to the Global Initiative for Asthma (GINA) guidelines. Asthma severity correlated with sputum eosinophilia and levels of eosinophil cationic protein. Despite treatment with high doses of ICS, eosinophilic inflammation was prominent. The authors conclude that the severity of persistent asthma is related to airway inflammation that continues despite treatment with ICS. These findings correspond with data from the current study, suggesting that there is wide variation of sputum eosinophilia in asthmatics, and that sputum eosinophilia can be a feature in patients treated with ICS. Possible mechanisms for the persistence of sputum eosinophilia despite treatment with ICS include under-treatment or overactive inflammation [1], the presence of inflammation in small airways inaccessible or poorly accessible to ICS [24], glucocorticoid resistance or reduced glucocorticoid receptor binding affinity [25], poor inhalation technique or noncompliance to medication [26], or unrecognised aggravating factors, such as nasal polyposis [27]. This suggests that measuring sputum eosinophils may provide useful additional information for disease monitoring.

The median age and duration of disease in the current study was found to be greater in the severe asthmatic group than the moderate and mild groups (table 1). The age of onset and sex was similar in all groups, indicating the main difference between the groups was age and duration of disease. Age was correlated with AR and sputum eosinophils, suggesting that degree of airway inflammation may be related to the subjects' age. Indeed, TEN BRINKE et al. [26], studying 132 subjects with chronic severe asthma, demonstrated that older age, longer asthma duration, persistent airway obstruction, and sputum eosinophilia were strongly associated. Multiple logistic regression analysis showed that only sputum eosinophils were associated with persistent airflow limitation. The authors concluded, "eosinophilic airway inflammation contributes to persistent airflow limitation in severe asthma" [26]. However, a recent study demonstrated no relationship between airway inflammation and lung function or airway hyperresponsiveness in mild ICS naïve asthmatics with disease duration $>16$ yrs, although the relationship was apparent when duration was $<16$ yrs [28]. Why severe-persistent asthma of a longer duration appears to be associated with sputum eosinophilia, yet mild non-ICS treated asthma of a longer duration appears not to be, is an interesting question. A possible explanation for this apparent paradox may be that the persistence of eosinophilic inflammation may lead to the development of severe disease despite treatment with ICS, although other factors, particularly airway remodelling [29] and autonomic dysfunction [27] are important in the pathophysiological deterioration in lung function that is associated with longer asthma duration [30].

This study utilised Kimura staining of sputum cytospins to determine apoptosis by light microscopy in sputum eosinophils. The use of Kimura staining as a measure of the presence of apoptotic eosinophils in suspension is a validated and sensitive technique [31]. The current study has demonstrated that Kimura is comparable to Diff-Quik for the assessment of eosinophil morphology by light-microscopic examination of sputum cytospins.

In summary, this study has demonstrated a relationship between sputum eosinophil apoptosis and clinical severity of chronic stable asthma measured by the Aas score, forced expiratory volume in one second, and self-reported symptom score. This study provides additional in vivo evidence that eosinophil apoptosis may play an important role in the resolution of asthmatic inflammation.

\section{References}

1. Bousquet J, Chanez P, Lacoste JY, et al. Eosinophilic inflammation in asthma. New Engl J Med 1990; 323: 10331035 .

2. Cohen JJ. Apoptosis: Mechanisms of life and death in the immune system. J Allergy Clin Immunol 1999; 103: 548-554.

3. Walsh GM. Eosinophil apoptosis: mechanisms and clinical relevance in asthmatic and allergic inflammation. Brit J Haematol 2000; 111: 61-67.

4. Vignola AM, Chanez P, Chiappara G, et al. Evaluation of apoptosis of eosinophils, macrophages and T lymphocytes in mucosal biopsy specimens of patients with asthma and chronic bronchitis. J Allergy Clin Immunol 1999; 103: 563573.

5. Woolley KL, Gibson PG, Carty K, Wilson AJ, Twaddell SH, Woolley MJ. Eosinophil apoptosis and the resolution of airway inflammation in asthma. Am J Resp Crit Care Med 1996; 154: 237-243.

6. Foresi A, Teodoro C, Leone C, et al. Eosinophil apoptosis in induced sputum from patients with seasonal allergic rhinitis and with asymptomatic and symptomatic asthma. Annals Asthma Allergy Immunol 2000; 84: 411-416.

7. Jang A-S, Choi I-S, Lee S, Seo J-P, Yang S-W, Park C-S. Bcl-2 expression in sputum eosinophils in patients with acute asthma. Thorax 2000; 55: 370-374.

8. Gibson PG, Saltos N, Fakes K. Acute anti-inflammatory effects of Budesonide in asthma. Am J Resp Crit Care Med 2001; 163: 32-36.

9. Bureau F, Seumois G, Jaspar F, et al. CD40 engagement enhances eosinophil survival through induction of cellular inhibitor of apoptosis protein 2 expression: Possible involvement in allergic inflammation. J Allergy Clin Immunol 2002; 110: 443-449.

10. Adachi T, Motojima S, Hirata A, Fukuda T, Makino S. Eosinophil viability-enhancing activity in sputum from patients with bronchial asthma, Contributions of interleukin-5 and granulocyte/macrophage colony-stimulating factor. Am J Respir Crit Care Med 1995; 151: 618-623.

11. Standards for the diagnosis and care of patients with chronic pulmonary disease (COPD) and asthma. Am Rev Resp Dis 1987; 136: 225-244.

12. Jones PW, Quirk FH, Baveystock CM. The St. George's Respiratory Questionnaire. Respir Med 1991; 85: Suppl. 6, 25-31.

13. Aas K. Heterogeneity of bronchial asthma. Allergy 1981; 149: 911-921.

14. Bousquet J, Chanez P, Chanal I, Michel FB. Comparison between RAST and Pharmacia CAP system: a new automated specific IgE assay. J Allergy Clin Immunol 1990; 85: 1039-1043.

15. Pin I, Gibson PG, Kolendowicz R, Denburg JA, Hargreave FE, Dolovich J. Use of induced sputum cell counts to investigate airway inflammation in asthma. Thorax 1992; 47: 25-29.

16. Pavord ID, Pizzichini MMM, Pizzichini E, Hargreave FE. The use of induced sputum to investigate airway inflammation. Thorax 1997; 52: 498-501.

17. Stern M, Savill J, Haslett C. Human monocyte-derived macrophage phagocytosis of senescent eosinophils undergoing apoptosis: mediation by $\alpha \mathrm{v} \beta 3 / \mathrm{CD} 36 /$ thrombospondin recognition mechanism and lack of phlogistic response. $\mathrm{Am}$ J Pathol 1996; 149: 911-921.

18. Walsh GM, Sexton DW, Blaylock MG, Convery CM. Resting and cytokine-stimulated human small airway epithelial cells recognise and ingest apoptotic eosinophils. Blood 1999; 94: 2827-2835.

19. Simon H-U, Yousefi S, Shranz C, Schapowal A, Bachert C, Blaser K. Direct demonstration of delayed eosinophil apoptosis as a mechanism causing tissue eosinophilia. J Immunol 1997; 158: 3902-3908.

20. Uller L, Persson CGA, Källström L, Erjefält JS. Lung tissue 
eosinophils may be cleared through luminal entry rather than apoptosis. Am J Resp Crit Care Med 2001; 164: 1948-1956.

21. Wardlaw AJ. Molecular basis for selective eosinophil trafficking in asthma: A multistep paradigm. J Allergy Clin Immunol 1999; 104: 917-926.

22. Wardlaw AJ, Brightling C, Green R, Woltmann G, Pavord I. Eosinophils in asthma and other allergic diseases. Brit Med Bull 2000; 56: 985-1003.

23. Louis R, Lau LCK, Bron AO, Roldaan AC, Radermecker M, Djukanovic R. The relationship between airways inflammation and asthma severity. Am J Respir Crit Care Med 2000; 161: 9-16.

24. Hamid Q, Song Y, Kotsimbos TC, et al. Inflammation in small airways in asthma. J Allergy Clin Immunol 1997; 100: 44-51.

25. Hamid QA, Wenzel SE, Hauk PJ, et al. Increased glucocorticoid receptor-beta in airway cells of glucocorticoid-insensitive asthma. Am J Respir Crit Care Med 1999; 159: 1600-1604.

26. ten Brinke A, Zwinderman AH, Sterk PJ, Rabe KF, Bel EH.
Factors associated with persistent airflow limitation in severe asthma. Am J Respir Crit Care Med 2001; 164: 744-748.

27. Lamblin C, Gosset P, Salez F, et al. Eosinophilic airway inflammation in nasal polyposis. $J$ Allergy Clin Immunol 1999; 104: 85-92.

28. Gronke L, Kanniess F, Holz O, Jorres RA, Magnussen H. The relationship between airway hyper-responsiveness, markers of inflammation and lung function depends on the duration of the asthmatic disease. Clin Exp Allergy 2002; 32: $57-63$.

29. Haley KJ, Drazen JM. Inflammation and airway function in asthma: what you see is not necessarily what you get. $\mathrm{Am}$ J Resp Crit Care Med 1998; 157: 1-3.

30. Cassino C, Berger KI, Goldring RM, et al. Duration of asthma and physiologic outcomes in elderly non-smokers. Am J Respir Crit Care Med 2000; 162: 1423-1428.

31. Walsh GM, Dewson G, Wardlaw AJ, Levi-Schaffer F, Moqbel R. A comparative study of different methods for the assessment of apoptosis and necrosis in human eosinophils. J Immunol Methods 1998; 217: 153-163. 